\title{
Integrating Unnormalised Semi-Structured Data Sources
}

\author{
Sasivimol Kittivoravitkul and Peter M⿳⺈冂大 Brien \\ Department of Computing, Imperial College London, London SW7 2AZ \\ sk297@doc.ic.ac.uk, pjm@doc.ic.ac.uk \\ http://www.doc.ic.ac.uk/automed
}

\begin{abstract}
Semi-structured data sources, such as XML, HTML or CSV files, present special problems when performing data integration. In addition to the hierarchical structure of the semistructured data, the data integration must deal with the redundancy in semi-structured data, where the same fact may be repeated in a data source, but should map into a single fact in a global integrated schema. We term semi-structured data containing such redundancy as being an unnormalised data source, and we define a normal form for semi-structured data that may be used when defining global schemas. We introduce special functions to relate object identifiers used in the global data model to object identifiers in unnormalised data sources, and demonstrate how to use these functions in query processing, update processing and integration of these data sources.
\end{abstract}

\section{Introduction}

Areas of application development such as the WWW, electronic commerce, bioinformatics and other scientifi c disciplines, have led to a growing demand for data representations that support complex, nested and rapidly evolving structures. Often applications in these areas use a semistructured data (SSD) data model, such as XML, HTML or one of a variety of flat-fi le formats (including CSV and TSV). With the proliferation of distributed and heterogeneous SSD, there is a clear need for techniques to perform data integration over these SSD sources, and provide a global unifi ed view of the data.

One of the main tasks in data integration is to defi ne the mappings between individual data sources and the unifi ed global view of those sources. Two basic approaches for specifying this mapping are global-as-view (GAV) and local-as-view (LAV) [10]. The former approach defi nes the concepts in the global schema as views over the local source schemas whereas the latter approach defi nes the sources as views over the global schema. Recently, a new approach called both-as-view (BAV) [13] has been proposed that specifi es a bi-directional mapping between each source and the global schema. Such bi-directional mappings allow data and queries to be translated in either direction from the global schema to the sources, and vice versa. This is important, for example, when integrating data in peer-to-peer contexts [14]. The use of BAV has been investigated in the integration of structured data sources [11], and some work has been carried out on integrating XML data sources $[12,20]$. The work on the XML integration has concentrated on specifying schema relationships and made strong assumptions about 


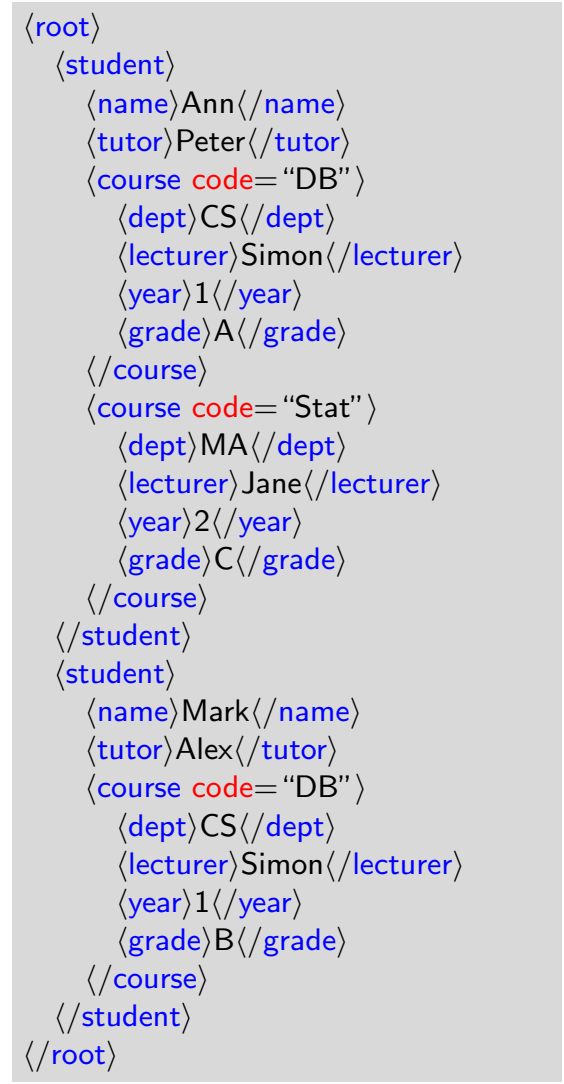

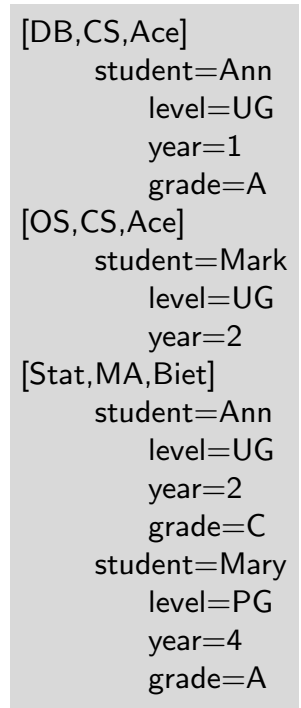

(b) $F_{2}$ Text file of students

(a) $F_{1}$ XML file of undergraduates

Fig. 1. Example unnormalised semistructured data sources

the data. In particular, they have assumed that there is no redundancy in the data to be integrated.

Fig. 1(a) and 1(b) illustrate two SSD sources which both contain a degree of redundancy, and which overlap with each other. Since they contain redundancy we call them unnormalised SSD sources - more precisely we regard anything with less than the SSD equivalent of second normal form as unnormalised. Fig. 1(a) contains an XML fi le $F_{1}$ with details of undergraduate students, where each course a student is sitting is placed within the student element. For each course, there is a record of the department that manages the course, the lecturer of that course, and the year of study and grade that the student has achieved in the course. Note that there is redundancy in this SSD, since the fact that the CS department runs the DB lectured by Simon is repeated for the two occurrences of that course.

Fig. 1(b) illustrates a structured text fi le $F_{2}$ containing information of courses taken by undergraduate and postgraduate students. The information in Fig. 1(b) is similar to that of the source in Fig. 1(a), but it is structured in different way i.e. student information 
is nested within course information, and provided information about the department building, which is not in $F_{1}$. It avoids the redundancy of $F_{1}$, in that the department of each course is only recorded once, but has its own redundancy in that the level of a student (UG or PG) is repeated for each course a student sits.

When integrating $F_{1}$ and $F_{2}$ we have to transform at least one of the files, and in particular deal with inverting the hierarchy present in one file to match that of the other. For example, if we choose in our global schema to model courses as containing multiple students (i.e. the $F_{2}$ view of the data) then when we transform $F_{1}$ we would want to have just one course department pair produced for each distinct code and dept pairing that exists in $F_{1}$ : i.e. produce a set of records containing just two courses, DB and Stat, with two students under the fi rst, and one under the second. Further, for each course, we would only want to have one dept value, but maintain the multiple year and dept associated with each student.

In this paper, we extend the BAV approach to the integration of SSD sources by relaxing assumptions or conditions in the preliminary work i.e. allowing unnormalised SSD sources to be integrated. The highlight of our approach is a semistructured data model which includes the notion of key constraints, and a mechanism to deal with redundancy of the data that allows data to be correctly translated in both directions, that is from sources to the global schema and vice versa. In contrast, most previous work in semistructured data translation/transformation $[3,5,15]$ has focused on retrieving data from data sources to the global schema, and only defi ned a one way mapping where data can be migrated from source to global schemas, but not vice versa. Popa et al. [16, 17] proposed a framework that semi-automatically generates invertible mappings between a relational source and an equivalent nested XML schema. Their mappings are not strictly invertible, since when there is more than one source, the reverse mapping gives back not only an original data but also information acquired by other sources. To our knowledge, no previous work specifi cally deals with normalisation of SSD sources in the context of data integration. Also, the subject of invertible mappings in SSD integration context has not been fully addressed.

The paper is structured as follows. Section 2 reviews a SSD modelling language called YATTA we shall use for representing SSD data sources. We use the YATTA model as basis for defi ning normal forms of SSD in Section 3, based on the well known notions of normal forms in relational models. This notion of second normal form is used as a basis for specifying our mappings using the keys in Section 4. It should be noted that it is only the global schema that must obey the second normal form, and the sources may remain unnormalised if we want to take a simple union of data, or they must be in the SSD equivalent of fi rst normal form if actual data (as opposed to schema) integration is to take place. Section 5 shows how queries and updates are processed using this mapping approach. Our summary and conclusions are in Section 6.

\section{The YATTA Data Model}

We adopt the YAT for Transformation-based Approach (YATTA) model [2] to model SSD sources. YATTA is a variation of the YAT model [4] that has two levels of abstraction, called the schema level the data level. 


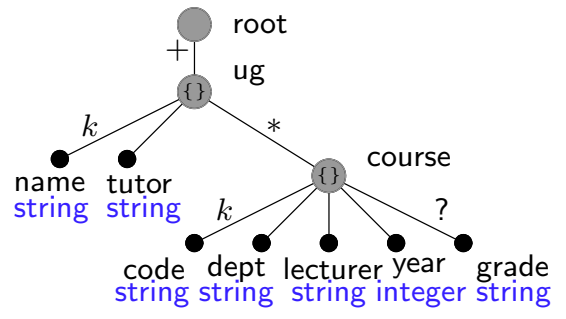

(a) YATTA schema $S_{1}$

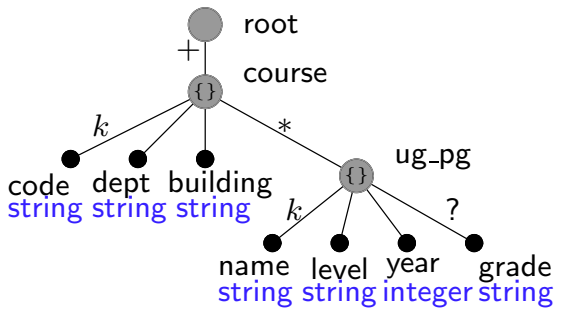

(b) YATTA schema $S_{2}$

Fig. 2. The YATTA schemas of the XML and text file Fig. 1(a) and 1(b)

A YATTA schema represents the structure of a SSD source. Each node in a YATTA schema is labelled with a pair of strings, representing its name and data type. The data type for a leaf node is one of the atomic types which are string, integer and real whereas the data type for a non-leaf node is of type list or set, represented by '[ ]' and ' \{\} '. Fig. 2(a) and 2(b) represent YATTA schemas for the XML and text fil les in Fig. 1(a) and 1(b).

Each YATTA schema edge between nodes $\langle i, j\rangle$ can be labelled with a cardinality constraint that determines the number of times corresponding nodes $j$ may occur under each node $i$ in a YATTA data tree, where ' $*$ ' indicates zero or more occurrences, ' + ' indicates one or more occurrences, '?' indicates zero or one occurrence, and label ' 1 ' indicates exactly one occurrence (and is implied if the edge is unlabelled). The symbol ' $k$ ' on an edge indicates that a $j$ is a key node, the values of which must be distinct from the values of other $j$ nodes that appear as siblings of $i$. Hence $k$ also implies the ' 1 ' constraint. In $S_{1}$, the key node of the course node is code, and says that each course for a particular ug will have a distinct code value.

Unlike a YATTA schema, a YATTA data tree has no labels on its edges. Each node is labelled by a tuple representing its name and value. The values of leaf nodes are the actual data in a data source whereas the values for non-leaf nodes are assigned by the system using integer identifiers (denoted by ' $\&$ ' followed by a number e.g. ' $\& 0$ '). The root node is always named root, and its identifi er differentiates between particular source fi les (such as $D_{1}$ ) that obey a schema (such as $S_{1}$ ).

Fig. 3(a) and 3(b) illustrate YATTA data trees of the XML and text fi les, and which match the YATTA schemas $S_{1}$ and $S_{2}$. For each data tree node there is a corresponding schema node with the same path, such that the data tree node value is compatible with the data type of that schema node. For example, the path $\langle\langle$ root, ug,course,grade $\rangle\rangle$ in $D_{1}$ leads to three grade data nodes. We will describe the extent of such nodes by listing their values along with the identifi ers of the parent, and hence $\langle\langle$ root, ug,course,grade $\rangle\rangle$ gives $\left[\left\{\& 3,{ }^{\prime} \mathrm{A}\right\},\left\{\& 4,{ }^{\prime} \mathrm{C}^{\prime}\right\},\left\{\& 5,{ }^{\prime} \mathrm{B}\right\}\right]$. In the schema, the same path leads to a simple string type node, which matches the type of the second value in each of the tuples in the extent list.

The occurrences of the data node follow the cardinality specifi ed by the symbols on the incoming edges of the corresponding node in the schema. For example, in Fig. 3(a), 


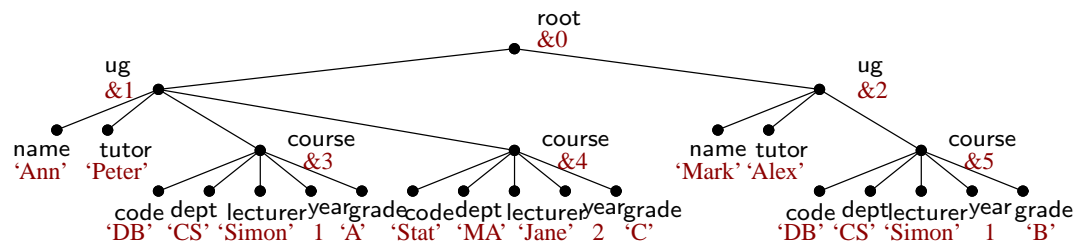

(a) $D_{1}$ The data tree of the XML file

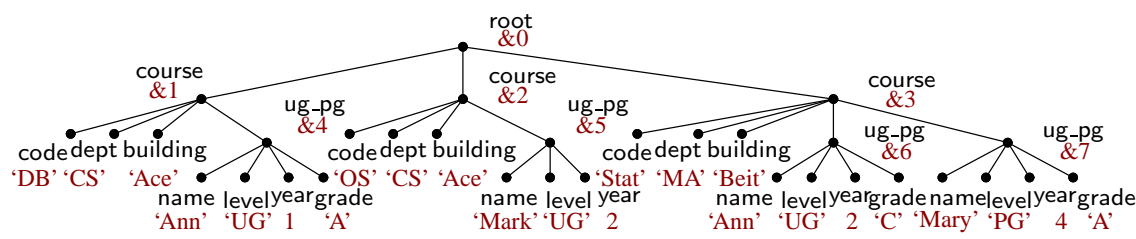

(b) $\mathrm{D}_{2}$ The data tree of the text file $F_{2}$

Fig. 3. Examples YATTA data trees for Fig. 1

each student has exactly one tutor, as specifi ed by implied ' 1 ' on the incoming edges of tutor in $S_{1}$, and each code of course only exists once for a particular undergraduate student which is also defi ned name as a key node. The label ' $k$ ' on the incoming edges of name and code in $S_{1}$ means the two ug nodes always have different name and no undergraduate student may take two courses with the same code.

\section{Normal Forms for Semistructured Data Sources}

In practice, many SSD sources have the property that each subrecord has a distinguishing attribute or set of attributes that uniquely identifi es the subrecord of a given record. We now formalise this idea into the notion of normal forms for SSD that may be used when defi ning global schemas to help avoid data redundancy, inconsistency and undesirable updating anomalies in the integration.

Normal forms have been extensively investigated in the relational model [6] and have recently been extended to SSD [1,8,19]. Both [1] and [8] defi ned a normal form for XML, called XNF, but the two approaches differ. [1] proposed the concept of functional dependency for XML, and defi ned BCNF for XML documents. In [8], an XML document is in XNF if its specifi cation does not contain potential redundancy w.r.t. a specifi ed set of constraints. Their defi nition is comparable to the requirement of $3 \mathrm{NF}$ in the relational model. [19] defi ned a normal form for SSD represented in the XML model, called NS-SS, which appears to be analogous to BCNF. In order to defi ne NSSS, they introduced the concept of 'extended functional dependency', which extends functional dependency in the relation model to support hierarchical data, and the notion of key constraints. 
The BCNF or $3 \mathrm{NF}$ proposed by $[1,8,19]$ gives a well-designed data source, but also increase the complexity in accessing a data source, and the use of SSD models is to achieve flexibility i.e. not too rigid design. Thus we work with weaker normal forms, comparable to first and second normal forms in the relational model, which are the minimum to achieve data integration.

In the relational model, first normal form (1NF) states that each attribute of a relation is functionally determined by its key value, and implies that each relation has a key, and that the non-key attributes take single values. We will defi ne 1NF in SSD as saying that each non-root, non-leaf node in the schema contains at least one key child leaf node (i.e. there is at least one $k$ beneath each non-leaf node), and that all leaf nodes do not use * or + cardinality constraints. This means that the non-key leaf nodes can be identifi ed by combining all the key nodes in the path to the non-key leaf node. Schema $\mathrm{S}_{1}$ obeys our $1 \mathrm{NF}$, and this means we can identify each ug and its tutor leaf node by name values, and we can identify each course and its dept, lecturer, year and grade leaf nodes, by the combination of its key node values i.e. code and name values. Note that in order to truely integrate data, as opposed to just the schema holding the data, sources must be in $1 \mathrm{NF}$, since that allows us to identify data values by a natural key (NK) (i.e. a set of values from the real world) rather than the artificial key (AK) (i.e. object identifi ers) used by the system.

In the relational model, second normal form (2NF) states that each attribute of a relation is functionally determined by primary key, but not by any proper subset of the key. In the YATTA model, this corresponds to the schema being in 1NF, with the additional constraint that all of the key nodes are necessary to determine each non-key node. $\mathrm{S}_{1}$ is not in $2 \mathrm{NF}$, since dept and lecturer are determined by code alone, and not code and name combined.

We can normalise schema $S_{1}$ to that shown in Fig. 4, by forming a copy of the course under the root, with just those non-key nodes which are determined by code alone being moved to the new course node, the remaining nodes staying as they were in $S_{1}$.

In this paper, we call sources not in the $2 \mathrm{NF}$, unnormalised data sources. As in the relational model, the redundancy in unnormalised SSD sources leads to diffi culties with updates, and in integration also leads to ineffi ciencies since the mapping tables based on the keys will contain redundant information. For example, to update the department for the 'DB' course from 'CS' to 'MA' in Fig. 3(a), we are faced with either the problem of searching the tree to fi nd every course containing 'DB' and 'CS' (and changing it) or the possibility of producing an inconsistent result, for example that the department for 'DB' might be given as 'MA' in one record and 'CS' in another. The next section explains how we write mapping rules that take account of this redundancy.

\section{Mapping Semistructured Data Sources Using Natural Keys}

The BAV approach integrates data sources by transforming source schemas into a global schema through sequences of transformations called pathways. Each transformation makes a 'delta change' to a schema, adding, deleting, or renaming a single schema node. Each transformation contains a query that specifi es the instances of a node in the 


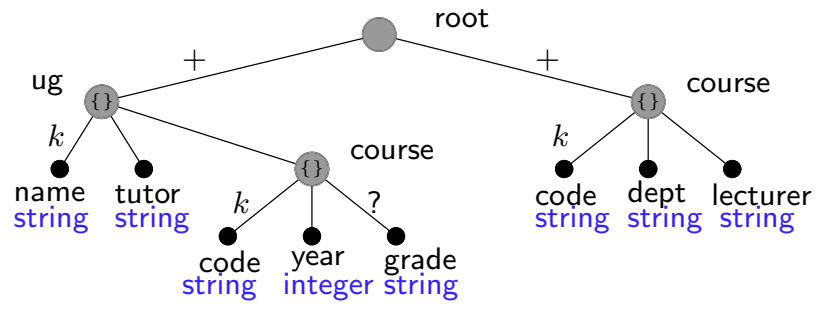

(a) $\mathrm{S}_{1}$ in $2 \mathrm{NF}$

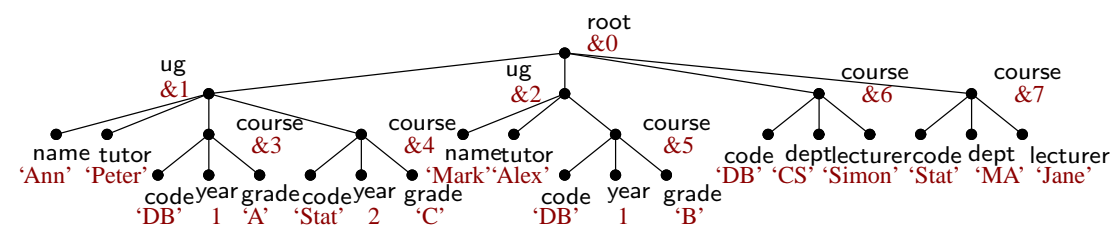

(b) The corresponding YATTA data tree

Fig. 4. The 2NF of YATTA source in Fig. 3(a)

corresponding data tree. We use the BAV transformation rules for the YATTA model that are defi ned in [2].

Suppose we want to integrate the 1NF SSD sources in Fig. 1(a) and 1(b). First we design a global schema $S_{g}$, such as that in Fig. 5, and then give mapping rules that defi ne how each node of a global schema can be defi ned from the source schemas. Based on the approach described in [2], when adding or deleting a node, we describe a scheme for the node which contains the pathway to the node, the type of the node, and the cardinality of the edge that leads to the node from its parent. For example, the scheme of the student node in $S_{\mathrm{g}}$ is $\langle\langle$ root,student,set, +$\rangle\rangle$ and the name is $\langle\langle$ root,student, name,string, $k\rangle\rangle$.

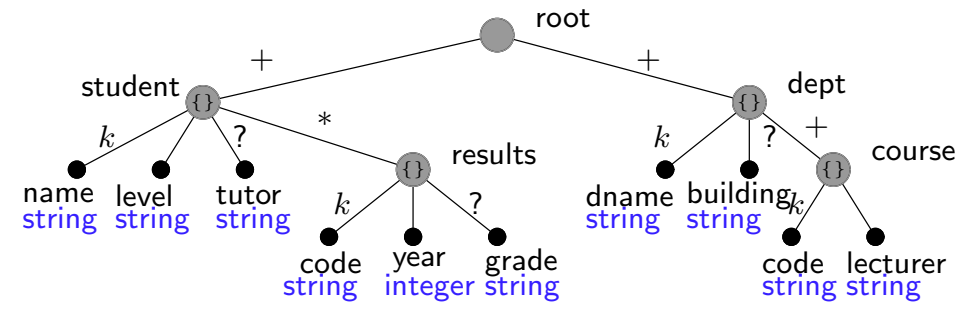

Fig. 5. The global schema $S_{g}$

The schemas $S_{1}$ and $S_{2}$ are transformed into $S_{g}$ by applying a pathway of YATTA transformation rules, where each pathway consists of a growth phase in which nodes in $S_{g}$ that do not exist in the source schema are added, followed by a shrinking phase 
in which nodes that exist in the source schema but not in $S_{g}$ are deleted. When a new node is added in the source schema, the query specifi es how the instances of a node in the corresponding data tree should be populated. When an existing node is deleted in a schema, the query specifi es how the instances of the node in the corresponding data tree can be restored from the remaining nodes. The mapping of the data sources to the global schema is therefore specifi ed through the queries in a pathway.

To ensure that source data is correctly mapped with the global schema, the transformations must take into account the following issues:

Identifier Conflicts Different data sources might be given different identifi ers for the nodes representing the same thing, and the same identifi er might be given for different things. For example, the identifi er ' $\& 1$ ' is assigned to the student node in $S_{1}$ in Fig. 3(a) and the course node in $S_{2}$ in Fig. 3(b). Hence, in specifying the mapping, a mechanism to resolve these conflicts is required.

Hierarchical structure The global schema might be structured in a different way from the source schemas, as shown in the examples. In this case, the mapping therefore involves preserving the relationships between data elements that are implied by the hierarchical structure of the data source.

To resolve identifi er conflicts, we apply the concept of a surrogate keys (SK) [7], which provides a way of mapping between a NK used in the real world, and an AK used by the system. This mapping is realised using two functions generateGID and generateSID, which are used in the queries of the add and delete transformations, respectively. The functions use the data values (NKs) to associate source identifi ers sids (AKs) with global identifi ers gids, which are generated as surrogates.

The generateGID function takes a source schema name, a sid, a list of data values and the name of a node that the transformation applied to. The function returns a new OID ( $g i d$ ) for every distinct list of data values, and returns the same gid for the same list of data values. Hence each gid serves as a surrogate for some set of data values. When a global schema is in $2 \mathrm{NF}$, these set of data values are the key values of the node that a transformation applied to.

Conversely, the generateSID takes a source schema name, a list of data values and the name of a node in the global schema. The function returns a source OIDs (sid) that have been used in generating gid for the same global schema node. The generateSID function can be thought of as the reverse of the generateGID function in the sense that generateGID takes sid in the source and generates gid for the global view whereas generateSID returns sid of the source.

For these functions to be applied in the pathway, the data sources and global schema must be at least in 1NF. This allows the key values in the different sources to be mapped to those of the global schema, thereby allowing data from those sources to be combined. If data sources or global schema are not in $1 \mathrm{NF}$, the integration will just take the union of the respective sources, which is not a real data integration.

To illustrate the generateGID and generateSID functions, we explain how they are used in the pathways $S_{1} \rightarrow S_{g}$ and $S_{2} \rightarrow S_{g}$, an extract from which is shown below. Note that $S_{1}$ and $S_{2}$ are in $1 \mathrm{NF}$ whereas $S_{\mathrm{g}}$ is in $2 \mathrm{NF}$ to avoid redundancy in the integrated data. The student node in $S_{g}$ is created by the add transformations (1) in $S_{1} \rightarrow S_{g}$ and (5) in $S_{2} \rightarrow S_{g}$. 


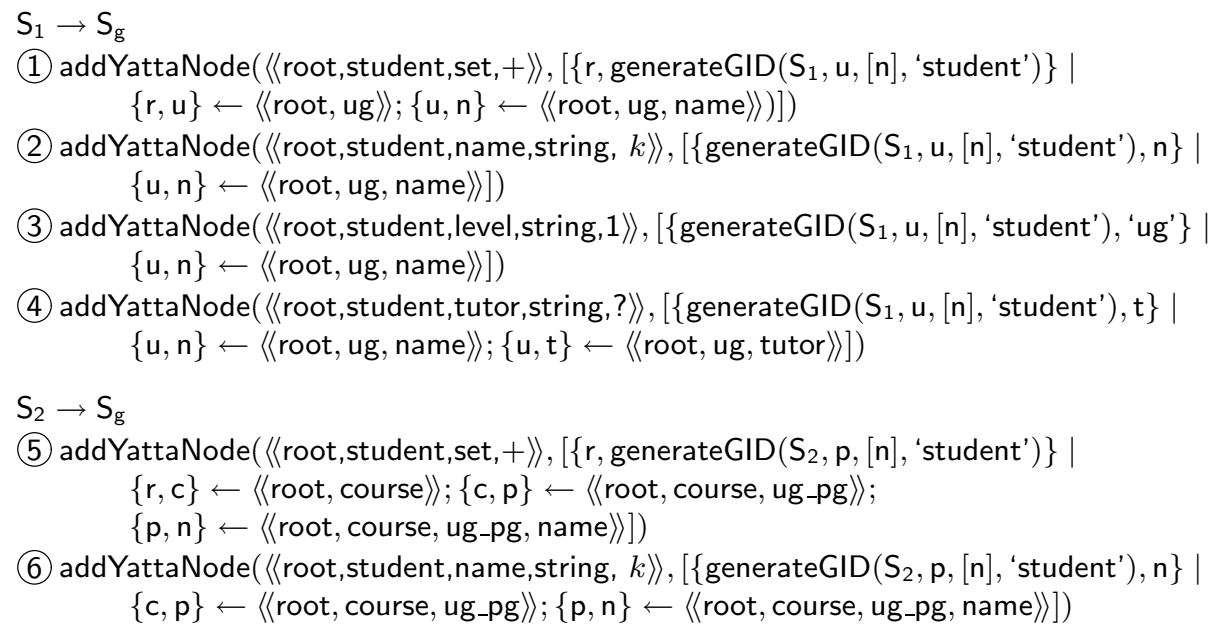

The IQL [18] query in (1) fi nds in the generator $\{r, u\} \leftarrow\langle\langle$ root, ug $\rangle$ the tuples $\{\& 0, \& 1\},\{\& 0, \& 2\}$, and $\{\mathrm{u}, \mathrm{n}\} \leftarrow\left\langle\langle\right.$ root, ug, name $\rangle$ the tuples $\left\{\& 1,{ }^{\prime}\right.$ Ann' $\},\{\& 2$, 'Mark' $\}$. Then the generateGID function is called with $\left(\mathrm{S}_{1}, \& 1,[\mathrm{Ann}]\right.$,'student') and $\left(\mathrm{S}_{1}, \& 2\right.$,[Mark],'student'). The function generates $\& 101$ and \&102 as new global integer identifi ers (gids) for each list of the data value, ['Ann'] and ['Mark'], which are the key values of student in $S_{g}$. Hence the list $[\{\& 0, \& 101\},\{\& 0, \& 102\}]$ will be associated with $\langle\langle$ root,student $\rangle\rangle$. A similar analysis for transformation (2) will give list $\left[\left\{\& 101,{ }^{\prime} A n n '\right\},\{\& 102, ' M a r k '\}\right]$ being associated with $\langle\langle$ root,student, name $\rangle$, and so on for the remaining transformations. The mapping of sids to gids for the student node through the values of the name node, which is the key node of student, is shown in Fig. 6 (though at this stage, the $S_{2}$ part of the graph should be ignored).

Similarly, the query in transformation (5) fi nds the tuples: $\{\& 0, \& 1\},\{\& 0, \& 2\}$, $\{\& 0, \& 3\}$ from $\{$ r,c $\} \leftarrow\langle\langle$ root, course $\rangle$, then the tuples $\{\& 1, \& 4\},\{\& 2, \& 5\},\{\& 3$, $\& 6\},\{\& 3, \& 7\}$ from $\{\mathrm{c}, \mathrm{p}\} \leftarrow\langle\langle$ root, course, ug_pg $\rangle$, and fi nally the tuples $\{\& 4$, 'Ann' $\}$, $\{\& 5, ' M a r k '\},\left\{\& 6,{ }^{\prime}\right.$ Ann' $\},\{\& 7, ' M a r y '\}$ from $\{\mathrm{p}, \mathrm{n}\} \leftarrow\langle\langle$ root, course, ug_pg, name $\rangle$. This causes generateGID to receive $\left(\mathrm{S}_{2}, \& 4,[\mathrm{Ann}]\right.$,'student'), ( $\mathrm{S}_{2}, \& 5$,[Mark],'student'), $\left(\mathrm{S}_{2}, \& 6,[\mathrm{Ann}]\right.$,'student') and $\left(\mathrm{S}_{2}, \& 7\right.$, , Mary],'student'). Since the gids for [Ann] and [Mark] already exist, the function returns $\& 101$ and $\& 102$ and generates a new gid $\& 103$ for [Mary]. Transformation (5) gives a list $[\{\& 0, \& 101\},\{\& 0, \& 102\},\{\& 0, \& 103\}]$ being associated with the scheme $\langle\langle$ root,student $\rangle\rangle$.

As illustrated in Fig. 6, the generateGID function groups together the ug and ug_pg nodes related to the same name, and creates a gid for each group. This resolves the identifi er conflicts among data sources and minimises the data redundancy in the integration. The data that is related to name such as tutor, is also put under the student node related to such name as specifi ed in the query in the transformation(4). The gids created by the generateGID function as well as its parameters can be stored as shown in Fig. 7 and 8.

Below are the transformations in the shrinking phase of $S_{1} \rightarrow S_{g}$ that remove the tutor, name and ug nodes in $S_{1}$ (we omit details of how before these transformations, grade, year, lecturer, dept, code and course are deleted). The generateSID function is 


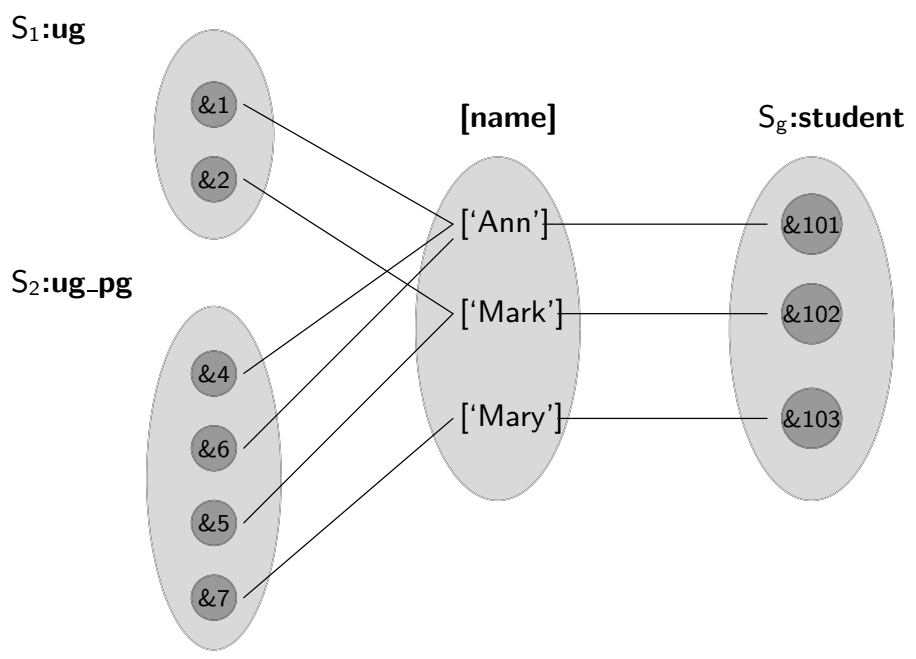

Fig. 6. The mapping between sids and gids of the student node

\begin{tabular}{c|c|l|l} 
local schema & sid & NK & name \\
\hline \hline $\mathrm{S}_{1}$ & [\&1 ] & ['Ann'] & student \\
$\mathrm{S}_{1}$ & {$[\& 2]$} & ['Mark'] & student \\
$\mathrm{S}_{2}$ & {$[\& 4]$} & ['Ann'] & student \\
$\mathrm{S}_{2}$ & {$[\& 5]$} & ['Mark'] & student \\
$\mathrm{S}_{2}$ & {$[\& 6]$} & ['Ann'] & student \\
$\mathrm{S}_{2}$ & {$[\& 7]$} & ['Mary'] & student \\
&. & .' &.
\end{tabular}

Fig. 7. sids and the data values

\begin{tabular}{c|c|c} 
gid & NK & name \\
\hline \hline$[\& 101]$ & [ 'Ann'] & student \\
{$[\& 102]$} & [ 'Mark'] & student \\
{$[\& 103]$} & [ 'Mary' ] & student \\
. &. &. \\
. &. &. \\
. &. &. \\
. &. &.
\end{tabular}

Fig. 8. gids and the data values

applied in this phase to allow the reverse transformation to recover the original data. This reversibility ensures information preservation in the transformation. Importantly, it allows data, queries and updates to be automatically migrated or translated in either direction between source and the global schemas.

$\mathrm{S}_{1} \rightarrow \mathrm{S}_{\mathrm{g}}$

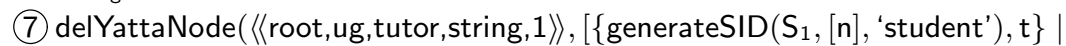
$\{\mathrm{s}, \mathrm{n}\} \leftarrow\langle\langle$ root, student, name $\rangle\rangle ;\{\mathrm{s}, \mathrm{t}\} \leftarrow\langle\langle$ root, student, tutor $\rangle\rangle ;$

$\{\mathrm{s}$, 'ug' $\} \leftarrow\langle\langle$ root, student, level $\rangle]])$

(8) delYattaNode( $\langle\langle$ root, ug, name,string, $k\rangle\rangle,\left[\right.$ \{generateSID $\left(\mathrm{S}_{1},[\mathrm{n}]\right.$, 'student'), $\left.\mathrm{n}\right\} \mid$ $\{\mathrm{s}, \mathrm{n}\} \leftarrow\langle\langle$ root, student, name $\rangle\rangle ;\left\{\mathrm{s},{ }^{\prime}\right.$ 'ug' $\} \leftarrow\langle\langle$ root, student, level $\left.\left.\rangle\rangle\right]\right)$

(9) delYattaNode( $\langle\langle$ root, ug, set, +$\rangle\rangle,\left[\left\{r\right.\right.$, generateSID $\left(S_{1},[n]\right.$, 'student') $\} \mid$ $\{\mathrm{r}, \mathrm{s}\} \leftarrow\langle\langle$ root, student $\rangle\rangle ;\{\mathrm{s}, \mathrm{n}\} \leftarrow\langle\langle$ root, student, name $\rangle\rangle ;$

$\{\mathrm{s}$, 'ug' $\} \leftarrow\langle\langle$ root, student, level $\rangle]])$

Transformation (7) removes the tutor node from ug. The query in the transformation states that the values of tutor in $S_{1}$ can be restored from tutor in $S_{g}$. It fi nds the tuples 
$\{\& 101$, 'Ann' $\},\{\& 102$, 'Mark' $\},\{\& 103$, 'Mary' $\}$ from $\{\mathrm{s}, \mathrm{n}\} \leftarrow\langle\langle$ root, student, name $\rangle$, then the tuples $\{\& 101$, 'Peter' $\},\{\& 102$, 'Alex' $\}$ from $\{\mathrm{s}, \mathrm{t}\} \leftarrow\langle\langle$ root, student, tutor $\rangle\rangle$, then the tuples $\{\& 101$, 'ug' $\},\{\& 102$, 'ug' $\}$ from $\{\mathrm{s}$, 'ug' $\} \leftarrow\langle\langle$ root, student, level $\rangle$. The generateSID function is called with $\left(\mathrm{S}_{1},\left[{ }^{\prime}\right.\right.$ Ann'], 'student') and $\left(\mathrm{S}_{1},[\right.$ 'Mark'],'student'). The function then looks up the key values in the table of Fig. 7, and restores the values of the ug node in $\mathrm{S}_{1}$ with $\& 1$ and $\& 2$, which are the sids related to 'Ann' and 'Mark' in the source $S_{1}$. The queries in (8) and (9) can be read in a similar manner.

\section{Queries and Updates over the Mapping}

After defi ning the mappings of data sources to the global schema, one may want to query or update data sources through the global schema. The bi-directional mappings allow queries and updates posed on the global schema to be automatically translated to ones poses on data sources.

\subsection{Query Translation}

To translate a query $Q_{g}$ posed on the global schema to a query on data source $S_{x}$, we need only consider delete transformations in the pathway $S_{g} \rightarrow S_{x}$. Every deleted construct appearing in the query $Q_{g}$ are substituted by the query in the transformations. For example, suppose we pose the query $Q_{1}$ on $\mathrm{S}_{\mathrm{g}}$ asking for all students, which in the IQL would take the form:

$$
Q_{1}=[\{x, y\} \mid\{x, y\} \leftarrow\langle\langle\text { root, student, name }\rangle\rangle]
$$

The pathways $\mathrm{S}_{\mathrm{g}} \rightarrow \mathrm{S}_{1}$ and $\mathrm{S}_{\mathrm{g}} \rightarrow \mathrm{S}_{2}$ can be automatically derived from $\mathrm{S}_{1} \rightarrow \mathrm{S}_{\mathrm{g}}$ and $S_{2} \rightarrow S_{g}$, respectively, by replacing delete for add, and replacing add for delete. Below are the inverse steps (2-1) in transformations (1)-(4), and 6-5 in transformations (5)-6).

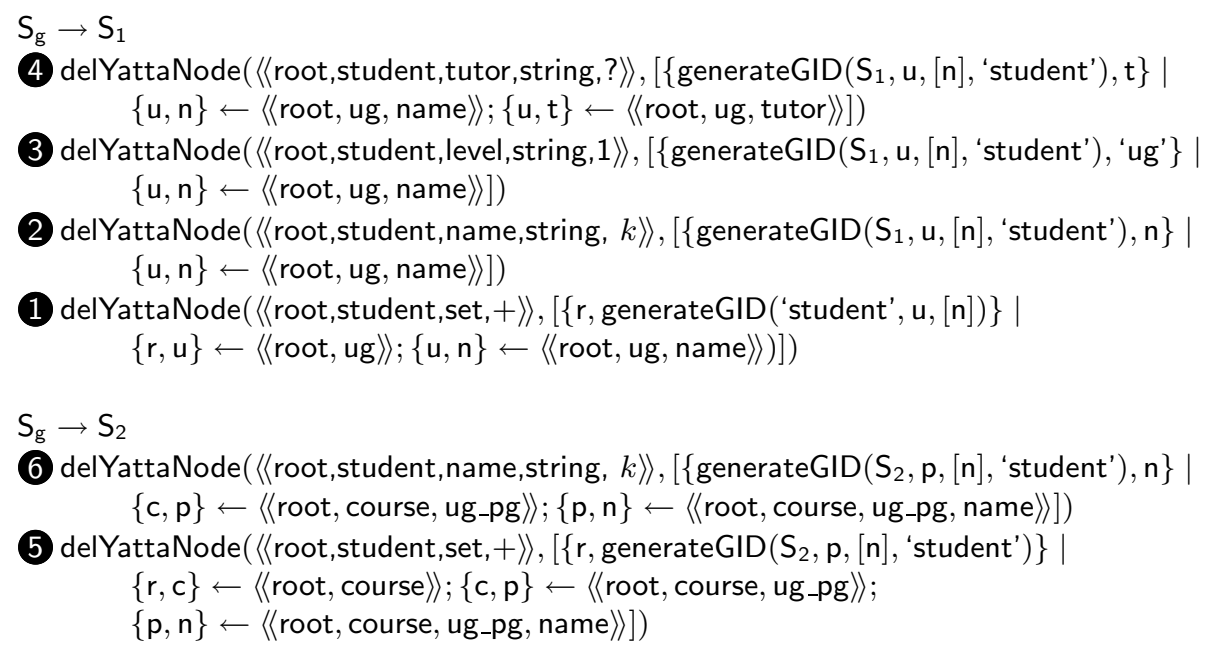


To translate the query $Q_{1}$ into source ones, the construct $\langle\langle$ root, student, name $\rangle$ is replaced by the queries $q_{1}$ and $q_{2}$ in transformations 2 in the pathway $\mathrm{S}_{1} \rightarrow \mathrm{S}_{\mathrm{g}}$ and 6 in the pathway $S_{2} \rightarrow S_{g}$, combined by the OR operator [9].

$$
Q_{1}=\left[\{x, y\} \mid\{x, y\} \leftarrow\left(q_{1} \text { OR } q_{2}\right)\right]
$$

The query $q_{1}$ returns a list $[\{\& 101$, 'Ann' $\},\{\& 102$, 'Mark' $\}]$ whereas the query $q_{2}$ gives a list $\left[\{\& 101, ' A n n '\},\left\{\& 102,{ }^{\prime} M a r k '\right\},\{\& 103, ' M a r y '\}\right]$ as described in the previous section. Using union semantics for the OR operator, we therefore get are all students from both data sources:

$$
Q_{1}=[\{\& 101, \text { 'Ann' }\},\{\& 102, \text { 'Mark' }\},\{\& 103, \text { 'Mary' }\}]
$$

In this simple example, the generateGID function reduces the redundancy by ensuring that 'Ann' is returned only once. Without the generateGID function, the name 'Ann' would appear three times as a result of different identifi ers of student nodes in the data sources.

In general, the generateGID function combines data that requires merging but has different identifi ers in the sources (e.g. student nodes with identifi ers $\& 2$ and $\& 5$, which are related to 'Mark'), and avoids combining distinct data that has the same identifi er in different sources (e.g. course nodes with identifi ers \& 2 in $D_{1}$ and $D_{2}$, which are related to 'Mark' and 'OS', respectively). In addition, the function allows related information in different sources to be brought together by the key values. For example, the information about the department building and the lecturer are in different sources, but by posing the query $Q_{2}$ below on $\mathrm{S}_{\mathrm{g}}$,

$$
\begin{aligned}
Q_{2}= & {[\{x, y, z, w\} \mid\{x, y\} \leftarrow\langle\langle\text { root, dept, building }\rangle\rangle ;} \\
& \{x, c\} \leftarrow\langle\langle\text { root, dept, course }\rangle ; \\
& \{c, z\} \leftarrow\langle\text { root, dept, course, code }\rangle ; \\
& \{c, w\} \leftarrow\langle\langle\text { root, dept, course, lecturer }\rangle]]
\end{aligned}
$$

the query results in a list of the departments, the building names, the course codes, and the lectures for the courses as shown below, since they are joined by the natural keys identifying dept and course.

$$
\begin{aligned}
Q_{2}= & {[\{\text { 'CS', 'ACE', 'DB', 'Simon' }\},\{\text { 'CS', 'ACE', 'OS', 'Void' }\},} \\
& \text { 'MA', 'Beit', 'Stat', 'Jane' }\}]
\end{aligned}
$$

\subsection{Update translation}

Applying an update $U$ requires giving the scheme of the construct to be updated, and the new value of the construct tuple. For example, to change the tutor which is associated to student with identifer \&101 to 'Fred', the update statement can be written as:

$$
U_{1}=\text { update }(\langle\text { root, student, tutor }\rangle,\{\& 101, \text { 'Fred' }\})
$$

Translating updates posed on a global schema to ones on data sources is different from translating queries described earlier. In update translation, all delete rules in pathways $S_{x} \rightarrow S_{g}$ that use the scheme of the construct to be updated should be retrieved. For the example, only (7) in the pathway $\mathrm{S}_{1} \rightarrow \mathrm{S}_{\mathrm{g}}$ matches the criteria for $U_{1}$, since 
its IQL query contains 〈 root,student,tutor $\rangle$. The data source is then updated by processing the queries accompanying the delete transformation using the new value of the construct tuple. In the example, the query accompanying (7) in the pathway $S_{1} \rightarrow S_{g}$ is processed using the new value $\{\& 101$, 'Fred' $\}$. The association between the gid \&101 and sid $\& 1$ is obtained by the generateSID function. The value $\{\& 1$,'Peter' $\}$ in the $\langle\langle$ root,ug,tutor $\rangle\rangle$ of $S_{1}$ is then updated to $\{\& 1$, 'Fred' $\}$.

Note that there may be multiple source nodes to update for some update statements. For example, with the update on $\mathrm{S}_{\mathrm{g}}$ to change the department name of the course with value \&110 to 'Math' such as the following:

$$
U_{2}=\text { update }(\langle\langle\text { root, course, dept }\rangle\rangle,\{\& 110, \text { 'Math' }\})
$$

There would be potentially several data source nodes that would be returned when the rules contain $\langle\langle$ root,course,dept $\rangle\rangle$ are found.

\section{Summary and Conclusions}

In this paper we have considered the issue of normalisation of SSD as part of a data integration process. We defi ned a SSD fi rst normal form that allows us to identify data values in sources by the use of a natural key, and hence perform data (as opposed to just schema) integration. It is proposed that the global schema be in second normal form to allow updates to data sources to be made in a consistent manner, and to minimise the size of the mapping tables between source and global schema object identifi ers. The normal forms considered in this paper are weaker (and simpler) than those defi ned in the literature, but if required, our approach could easily be extended to use higher normal forms, such as those defi ned in [19]. However, 2NF is suffi cient for our approach to work, and provided a data source is not denormalised by the data integration rules (i.e. brought down from a higher normal form to $2 \mathrm{NF}$ ), then no update anomalies will be introduced by the process of data integration.

We introduced the functions generateGID and generateSID, which use a natural key to relate object identifi ers in the $1 \mathrm{NF}$ (or higher) source schemas to identifi ers in the global schema, and showed how these functions are used in the bi-directional transformation pathways of the BAV approach to data integration. The generateGID function allows data from different sources that require merging/joining to be correctly combined. It solves the identifi er conflicts among local data sources, mapping them to a single global schema identifi er, by relating them via a natural key. This reduces redundancy in the integrated schema, and allows updates to be performed without introducing anomalies. The generateSID function allows the original data sources to be restored. It ensures the pathways are reversible, and therefore allows updating from the global schema to the sources, and allows data and queries to be migrated in both directions - a functionality particularly important in peer-to-peer contexts.

Our approach has been implemented in the AutoMed data integration system (details of which may be found at (http://www.doc.ic.ac.uk/automed). The generateGID and generateSID functions have been integrated into the query processor to allow the system to correctly combine data from different sources, to support updating of data sources and to enable the original data sources to be restored. For the future work, 
we will be building larger case studies focusing on the integration of biological data sources, which are often held in the form of flat fi les, HTML or XML.

\section{References}

1. M. Arenas and L. Libkin. A normal form for xml documents. ACM Transactions on Database Systems, 29(1):195-232, 2004.

2. M. Boyd, S. Kittivoravitkul, C. Lazanitis, P.J. McBrien, and N. Rizopoulos. AutoMed: A BAV data integration system for heterogeneous data sources. In Proc. CAiSE2004, volume 3084 of $L N C S$, pages 82-97. Springer-Verlag, 2004.

3. V. Christophides, S. Cluet, and J. Siméon. On wrapping query languages and efficient xml integration. SIGMOD Rec., 29(2):141-152, 2000.

4. S. Cluet, C. Delobel, J. Siméon, and K. Smaga. Your mediators need data conversion! In Proc. SIGMOD'98, pages 177-188. ACM Press, 1998.

5. S. Cluet and J. Siméon. Data integration based on data conversion and restructuring. Technical report, Verso database group- INRIA, France, 1997.

6. C.J. Date. An Introduction to Database Systems. Addison-Wesley, 8th edition edition, 2004.

7. C.J. Date, H. Darwen, and D. McGoveran. Relational Database: Selected Writings 19941997. Addison-Wesley, 1998.

8. D.W. Embley and W.Y. Mok. Developing xml documents with guaranteed "good" properties. In Proc. 20th ER, pages 426-441, 2001.

9. E. Jasper, N. Tong, P.J. McBrien, and A. Poulovassilis. View generation and optimisation in the AutoMed data integration framework. In Proc. Baltic DB\&ISO4, volume 672 of Scientific Papers, pages 13-30. Univ. Latvia, 2004.

10. M. Lenzerini. Data integration: A theoretical perspective. In Proc. PODS'02, pages 233246. ACM, 2002.

11. P.J. McBrien and A. Poulovassilis. A uniform approach to inter-model transformations. In Proc. CAiSE'99, volume 1626 of LNCS, pages 333-348. Springer, 1999.

12. P.J. McBrien and A. Poulovassilis. A semantic approach to integrating XML and structured data sources. In Proc. CAiSE'01, volume 2068 of LNCS, pages 330-345. Springer, 2001.

13. P.J. McBrien and A. Poulovassilis. Data integration by bi-directional schema transformation rules. In Proc. ICDE'03, pages 227-238. IEEE, 2003.

14. P.J. McBrien and A. Poulovassilis. Defining peer-to-peer data integration using both as view rules. In Proc. DBISP2P, at VLDB'03, Berlin, Germany, 2003.

15. Y. Papakonstantinou, S. Abiteboul, and H. Garcia-Molina. Object fusion in mediator systems. In Proceedings of the 22th International Conference on Very Large Data Bases, pages 413-424. Morgan Kaufmann Publishers Inc., 1996.

16. L. Popa, Y. Velegrakis, R.J. Miller, M.A. Hernandez, and R. Fagin. Translating web data. In Proc 28th VLDB, pages 598-609, 2002.

17. L. Popa, Y. Velegrakis, R.J. Miller, M.A. Hernandez, and R. Fagin. Translating web data. Technical report, Department of Computer Science, University of Toronto, 2002.

18. A. Poulovassilis. The automed intermediate query language. Technical report, Department of Computer Science, Birkbeck College, 2001.

19. X. Wu, T.W. Ling, S.Y. Lee, M. Lee, and G. Dobbie. Nf-ss: A normal form for semistructured schema. In ER 2001 Workshops, pages 292-305, 2001.

20. L. Zamboulis and A. Poulovassilis. Using automed for $\mathrm{xml}$ data transformation and integration. In Z. Bellahsene and P.J. McBrien, editors, Proc. DIWeb04, CAiSE Workshop Proceedings Volume 3, pages 58-69, 2004. 